Meta

Journal des tradlucteurs

Translators' Journal

\title{
Collocations et langues de spécialité
}

\section{André Clas}

Volume 39, numéro 4, décembre 1994

Hommage à Bernard Quemada : termes et textes

URI : https://id.erudit.org/iderudit/002327ar

DOI : https://doi.org/10.7202/002327ar

Aller au sommaire du numéro

Éditeur(s)

Les Presses de l'Université de Montréal

ISSN

0026-0452 (imprimé)

1492-1421 (numérique)

Découvrir la revue

Citer cet article

Clas, A. (1994). Collocations et langues de spécialité. Meta, 39(4), 576-580.

https://doi.org/10.7202/002327ar d'utilisation que vous pouvez consulter en ligne.

https://apropos.erudit.org/fr/usagers/politique-dutilisation/ 


\section{COLLOCATIONS ET LANGUES DE SPÉCIALITÉ}

ANDRÉ Clas

Université de Montréal, Montréal, Canada

Nous avons tous l'impression que la liberté dans l'utilisation des mots est absolue, que les contraintes dans le choix des mots sont inexistantes et que nos possibilités sont sans limites. Nous sommes persuadés que nous pouvons utiliser les mots du langage courant comme il nous sied.

Et pourtant, des restrictions existent et la liberté de choix semble bien être une illusion, du moins partielle. La possibilité de choix n'existe qu'au moment où je choisis. Dès que mon premier choix est fait, la suite m'est d'une certaine façon imposée. Un premier mot ne se combine pas nécessairement à n'importe quel autre. En effet, la combinabilité, c'està-dire l'aptitude qu'ont les mots à se joindre aux autres mots, est limitée, bien plus, elle est souvent imposée. Nous sommes donc d'une certaine façon en «liberté de parole surveillée»! Ainsi, si je veux savoir quelque chose, je peux interroger, demander des renseignements ou poser des questions, mais je ne peux en aucun cas *demander une question, alors que l'anglais dit ask a question et l'allemand, eine Frage stellen.

La plupart du temps, le locuteur n'est pas conscient de ce type de restriction; ce qui ne se dit pas, il ne le dit pas, par habitude, parce qu'il «sait» ce qui se dit ou doit se dire. Ainsi, il ne dira jamais *le crayon souriait, sauf dans un contexte très ludique, de dessins animés, par exemple. Il y a donc des restrictions imposées par la sémantique aux objets du discours. Mais en réalité cette «confraternité sémantique» n'est pas un empêchement combinatoire. Disons que les mots n'ont pas la même autonomie les uns par rapport aux autres. Certains peuvent accepter plus facilement un plus grand nombre de «confrères», d'autres refusent toute association. Il suffit ici d'évoquer l'adjectif cochère ou saur pour s'en convaincre. Notons cependant que certaines restrictions peuvent être levées à cause de l'évolution des réalités: parler de «carton de lait», de «sac en plastique de lait», d'une «souris» d'ordinateur était inadmissible il y a encore quelques années, tout comme «voler de Paris à Montréal» était une utopie il y a quelques décennies encore. Les mots peuvent donc être compatibles, sans que leur sémantisme soit compatible à première vue. $\mathrm{Si}$, en français, on peut donner, asséner, flanquer un coup, mais porter un coup dur ou fatal, en allemand, on dira einen Schlag versetzen. Ces combinaisons ne sont pas prévisibles, c'està-dire sémantiquement motivées, la lexicographie se doit donc de les signaler. Comment en effet savoir que le coucou français, tout comme le rossignol, chante et que le coucou allemand appelle (der Kuckuck ruft; die Nachtigall schlägt)!

La traduction, l'enseignement ou plus globalement les comparaisons des langues obligent très souvent à une prise de conscience restrictive. Ce qui se dit d'une façon dans une langue, ne se dit pas forcément de la même façon dans les autres. Toute langue est une série d'habitudes: les «formules langagières» nous sont données. Bien sûr elles peuvent être plus ou moins riches, mais dans de nombreux de cas, elles sont contraignantes. Ainsi quelqu'un qui a «une bonne mémoire» a une mémoire d'éléphant, une mémoire prodigieuse, une mémoire excellente, une mémoire étonnante,... en français et ein gutes Gedächtnis, ein ausgezeichnetes Gedächtnis,... en allemand. La série est plus ou moins riche, mais elle est limitée. Si le français dit odeur nauséabonde et façon écceurante, l'anglais a comme 
correspondant a nauseating smell et a nauseating manner, et toute autre combinaison relèverait de la création stylistique, plus ou moins acceptable et plus ou moins acceptée.

Ce que nous appelons «formules langagières» correspond en fait à ce que l'on nomme traditionnellement «cooccurrent», «cooccurrence lexicale restreinte» (Mel'čuk 1984), «collocation» (Galisson 1976) ou «phraséologie (phraséologisme)». Les mots ainsi associés montrent une «affinité» entre eux qui limitent leurs possibilités combinatoires. Cette affinité élimine certaines autres combinaisons et restreint le nombre de sens possibles. La collocation se distingue de la combinaison libre par cette restriction, et de la combinaison figée (phrasème) par sa caractéristique de transparence. Ainsi acheter un chapeau n'est pas une collocation, car le sens de acheter ou de chapeau n'est en rien restreint ou limité. Il n'y a aucune affinité entre ces mots, puisqu'on peut acheter énormément de choses et même des personnes. La collocation est encore une unité de la langue (et, répétons-le, doit donc être enseignée et notée dans les dictionnaires) et non de la parole, au sens saussurien des mots, tout comme le phrasème, mais à la différence de ce dernier, la collocation est toujours transparente: le sens global est déductible des unités de composition, l'un caractérisant l'autre. Si joie débordante, folle joie (allemand, überschäumende Freude), jour faste (allemand, Glückstag), jour funeste, jour néfaste (allemand, Unglückstag, schwarzer Tag) sont des collocations, perdre les pédales (= perdre ses moyens de réagir), rat de cave (= bougie), tirer son chapeau ou tirer sa révérence (= mourir, en anglais to kick the bucket), acheter chat en poche (allemand, die Katze im Sack kaufen) sont des phraséologismes, car le sens final n'est pas déductible du sens des composants, il n'y a aucune transparence.

Pour éviter toute mauvaise interprétation, rappelons que pour qu'il y ait collocation, il faut que les mots (le caractérisant (le collocatif ou colloquant) et le caractérisé (la base ou le collocateur) aient une affinité de sens sans que la combinatoire des unités ne modifie les caractères sémantiques essentiels de ces unités.

Toutes ces considérations ne sont nullement neuves. Déjà Arsène Darmesteter (1887: 107) écrivait que : «Les mots ne vivent pas isolés, dans notre pensée et sur nos lèvres. Ils sont en commerce réciproque les uns avec les autres, parce que représentants de nos idées, ils reproduisent dans la combinaison de la phrase le mouvement de la pensée avec toute la complexité des faits intellectuels qui la constituent. Considérée à ce point de vue, l'étude des mots soulève une nouvelle question, celle des actions diverses qu'ils peuvent subir de la part les uns des autres.» Et Aurélien Sauvageot (1964: 69) notait qu': «En dehors de ces quelques cas extrêmes, il existe une énorme partie du vocabulaire qui est constituée de mots qui n'ont qu'un rayon d'action limité. Ces vocables ne se construisent qu'avec un nombre plus ou moins réduit d'autres mots, toujours les mêmes, avec lesquels ils forment des locutions stéréotypées.» Sauvageot cite toute une série de «clichés»: perpétrer un crime, un forfait, une mauvaise action; commettre une faute, un crime, un péché, une mauvaise action, une erreur; élever, émettre une protestation; prononcer un discours, une allocution; proférer une parole (des paroles), une injure, une plainte; effectuer une opération, un calcul, un voyage; toucher, percevoir un traitement, une solde, des émoluments, des honoraires.

On peut admettre le terme de «cliché» utilisé par Sauvageot, à condition de lui retirer toute connotation péjorative, même si certaines collocations sont hyperutilisées. Peut-on (ou faut-il) dire autre chose qu'aberration mentale ou adieu déchirant, poignant, ou touchant, ou prendre, emprunter un raccourci, avoir la nostalgie ou garder la nostalgie, jeter la panique, semer la panique, être pris de panique, être gagné par la panique, être en panne, avoir une panne, tomber en panne, rester en panne?

Nous sommes bien en mesure de nommer les différentes réalités et la connaissance des règles grammaticales nous guide pour exprimer les messages voulus, mais rien ne nous permet de savoir quelles «combinaisons de mots» sont utilisables et à utiliser ou 
«quel est le rayon d'action», pour reprendre le mot de Sauvageot, des divers mots. Le cliché n'est qu'une combinaison habituelle, très (trop) fréquente (mais avons-nous, dans beaucoup de cas, tellement le choix ?), mais uniquement pour ceux qui savent!

Si la constatation du phénomène de la collocation est quelque peu générale, de même que des relevés d'exemples sont nombreux, la théorisation, elle, reste beaucoup plus parcimonieuse et plus difficile à élaborer. On peut répartir banalement les collocations en divers groupes basés sur une fonction syntagmatique, soit:

1) verbe et nom, où le verbe a un contenu sémantique très général proche simplement de «faire» (prononcer un discours);

2) nom et adjectif (rude épreuve, marque distinctive);

3) adverbe et adjectif (vachement bon);

4) verbe et adverbe (boire goulument);

5) nom (sujet) et verbe (la cloche sonne, le chat miaule, l'abeille bourdonne);

6) marquage de la quantité (unité ou collectif) du nom (essaim d'abeilles, troupeau de vaches, pincée de sel, barre de chocolat).

La première catégorie (verbe et nom) est relativement facile à déterminer puisque le verbe, contrairement à la tendance habituelle où il est un parfait «sémantisme», doit avoir un contenu sémantique vide ou presque, c'est-à-dire être un verbe avec une simple fonction d'«opérateur», et le nom contient tout le sémantisme. Bien entendu, certains noms peuvent se combiner avec un ou plusieurs collocatifs différents. Par contre, la catégorie «nom et adjectif» est déjà beaucoup plus riche en combinaisons. Très souvent, la combinabilité est importante et ne se dégage réellement ou n'apparaît pertinente que par comparaison avec d'autres langues. Les catégories «adverbiales» semblent extrêmement limitées et frisent la banalité du cliché puisque les adverbes sont très souvent les mêmes (grande, immense importance). Quant au groupe «sujet verbe», on peut en fait distinguer deux groupes: le cri ou mouvement caractéristique des animaux où il n'y a souvent qu'un seul verbe particulier (le chien aboie), et le verbe descriptif de la situation (la tour se dresse sur la colline). La catégorie qui décrit l'unité d'une masse ou le collectif est relativement limitée, ce sont les «composés» formés avec des mots comme peloton, poignée, meute, harde, harpail, grain, grêlon, etc.

Ce sont ces mêmes catégories de collocations que l'on retrouve chez Igor Mel'čuk (1984) qui les répartit en plus de 21 variétés sémantico-syntaxiques qu'il qualifie de «fonctions lexicales». Il note, par exemple, les fonctions lexicales suivantes:

Sing (un quantum régulier de...), Sing(riz) = un grain [de riz]

Mult (ensemble régulier de...), Mult(chien) $=$ meute

Culm (culmination de...), Culm(joie) = comble [de la joie] ;

Magn (très, intense, à un degré élevé...), Magn(désir) = ardent

Plus, Minus (plus, moins; fonctions lexicales qui se combinent obligatoirement avec d'autres fonctions lexicales), IncepPredMinus (vitesse) = diminuer, décroître

Ver (tel que cela doit être), Ver(peur) = justifiée

Bon (louange codifiée), Bon(conseil) = précieux

Pos (évaluation positive des actants), $\operatorname{Pos}_{2}$ (opinion) = favorable, avantageuse

Pred (verbalisation des fonctions adjectivales; la fonction lexicale se combine toujours avec d'autres fonctions), IncepPredMinus(vent); se calmer, mollir

Oper (verbe qui accompagne le nom des divers actants), Oper $_{1}$ (attention) $=$ faire ; $\mathrm{Oper}_{2}$ (attention) $=$ attirer

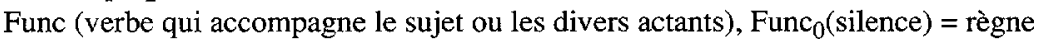

Labor (verbe qui accompagne le sujet ou les divers actants), Labor ${ }_{12}$ (soin) $=$ entourer [quelqu'un de soins] 
Involv (verbe qui accompagne le sujet), Involv(tempête) = balayer

Incep, Cont, Fin (commencer, continuer, cesser; ces fonctions lexicales s'utilisent le plus souvent en combinaison avec d'autres), IncepOper $_{1}$ (influence) $=$ garder, FinOper ${ }_{1}$ (influence) $=$ perdre, FinFonc $c_{0}$ (vent $)=$ se calmer

Caus, Liqu, Perm (causer, liquider, permettre ; ces fonctions s'emploient le plus souvent en combinaison avec d'autres), CausOper ${ }_{1}$ (désespoir) = pousser, réduire [quelqu'un au

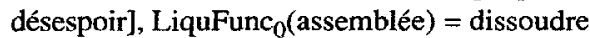

Real(réaliser), $\operatorname{Real}_{1}$ (piège) $=$ tendre

Fact(se réaliser), Fact(soupçon) $=$ se confirmer

Labreal(réaliser), Labreal(mémoire) $=$ conserver [quelque chose en mémoire]

Manif(se manifester, se combine avec d'autres fonctions), Manif(joie) = jaillir, éclater;

Caus $_{1}$ Manif(exigence $)=$ formuler

Degrad(se dégrader), Degrad(lait) $=$ tourner

Excess(de façon excessive), Excess(moteur) = s'emballer

La classification de ces fonctions lexicales est obtenue en s'appuyant à la fois sur un contenu sémantique et une fonction grammaticale. Ces fonctions permettent de rendre compte avec précision des valeurs sémantiques et grammaticales des collocations. Sans doute la tâche classificatrice est longue et parfois délicate à manier, mais elle devient une nécessité si l'on veut comprendre et faire comprendre les affinités des mots, et les faire utiliser correctement par une machine, par exemple, qui génère un texte. Il faut noter que Mel'čuk intègre lui aussi la cooccurrence de substantifs avec d'autres substantifs dans ses fonctions lexicales (Sing, Mult, Culm).

Bien entendu, la richesse de combinabilité par affinité est variable en fonction des mots, on peut se demander quels mots sont à retenir. En fait, comment choisir et distinguer les collocations des combinaisons plus libres? On peut encore se demander si les domaines des langues de spécialité connaissent les mêmes restrictions d'usage et quelles sont les possibilités collocatives puisque, par définition, le domaine d'emploi des mots techniques (les terminologismes) est le plus restreint.

Pour répondre à ces questions, nous allons examiner quelques verbes relevés dans le Dictionnaire des matières plastiques (1966). On peut trouver, pour la lettre A, les exemples suivants:

abaisser (le point de fusion); abraser (un métal); absorber (un effort mécanique); absorber (l'eau); accélérer (la résine); accroître (le dosage); aciduler (une solution); activer (une combinaison); initier (une polymérisation); affaiblir (les qualités); ajuster (un appareil); alcooliser (une solution); alcoyler (un métal); amarrer (les éprouvettes); améliorer (les textiles); amorcer (la réaction); appliquer (une pression); appliquer (une couche); arrêter (l'écoulement); aspirer (le gaz); anténuer (les tensions); aviver (les bords).

L'analyse donne les collocations suivantes:

abaisser = faire en sorte que le point de fusion soit moindre, ce qui s'exprime dans le système formalisé de Mel'čuk comme : CausPredMinus(point de fusion) = abaisser, absorber $=$ quelque chose fait que l'effort mécanique est «fait», soit la fonction lexicale : Fact $_{2}$ (effort mécanique) $=$ absorber.

accélérer = quelque chose fait que la résine se fige plus rapidement, soit la fonction lexicale : CausPlusFact $_{0}($ résine $)=$ accélérer .

accroître = quelque chose (quelqu'un) fait en sorte que le dosage soit plus élevé, soit la fonction lexicale : CausPredPIus(dosage) $=$ accroître.

activer = quelque chose (quelqu'un) fait en sorte que la combinaison soit plus rapide, soit la

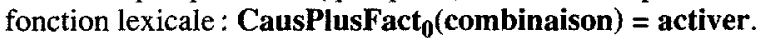

initier = quelque chose fait que la polymérisation commence, soit la fonction lexicale: CausFunc(polymérisation) $=$ initier . 
affaiblir = quelque chose fait que les qualités deviennent moindres, soit la fonction lexicale : CausPredMinus(qualités) = affaiblir.

amarrer = quelqu'un fait que les éprouvettes soient comme il faut, soit la fonction lexicale : CausFact $_{0}$ (éprouvettes) $=$ amarrer.

améliorer $=$ quelque chose (quelqu'un) fait que les textiles soient encore mieux, soit la fonction: CausPredPlusBon(textiles) $=$ améliorer .

amorcer $=$ quelque chose fait que la réaction commence, soit la fonction lexicale : CausFunc(réaction) $=$ amorcer.

appliquer $=$ faire en sorte que la pression ait lieu, soit la fonction : $\mathbf{O p e r}_{1}(\mathbf{p r e s s i o n})=$ appliquer.

appliquer $=$ quelque chose fait que la couche soit mise, soit la fonction lexicale : Caus $_{1}$ Func $_{2}($ couche $)=$ appliquer.

arrêter = quelque chose fait que l'écoulement ne soit plus, soit la fonction lexicale :

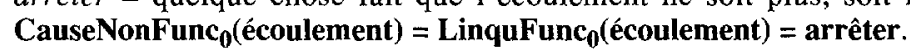

atténuer = quelqu'un fait que les tensions soient moindres, soit la fonction lexicale : CausPredMinus(tensions) $=$ atténuer.

aviver = quelqu'un fait que les bords soient tels qu'ils doivent être, soit la fonction lexicale : CausPred Ver = aviver.

Les autres exemples, soit abraser (un métal), absorber (l'eau), aciduler (une solution), alcooliser (une solution), alcoyler (un métal), aspirer (les gaz) sont des cooccurrences libres. En effet, les verbes sont soit des verbes qui peuvent accepter $n$ 'importe quel complément, la série des acceptations est extrêmement large (abraser une pierre, un nodule, la peau...; absorber de l'eau, de la lumière, des rayons, des économies...; aspirer un liquide, une poudre, un objet...), ou encore les verbes ont un sémantisme plein (aciduler, alcooliser, alcoyler).

Certains peuvent se demander quel est l'intérêt d'un tel «exercice». Rappelons que Roman Jakobson (1971:261) affirmait que le sens d'un signe linguistique est sa traduction dans un autre signe. En fait, pour exprimer la même idée, ou pour bien traduire, chacun sait qu'il faut décomposer la signification, la déverbaliser d'une certaine façon, pour ensuite reconstruire le sens d'un autre façon ou dans une autre langue. Cet exercice n'est rien d'autre. En plus, il n'est pas inutile de rappeler que de telles entreprises descriptives permettent de mieux comprendre le fonctionnement de la langue et de prévoir des outils utiles pour «générer» automatiquement des textes ou pour traduire automatiquement certains textes.

\section{RÉFÉRENCES}

DARMESTETER, Arsène (1887) : La Vie des mots étudiée dans leurs significations, Paris, Éditions Champ Libre, réimpression de 1979.

HAUSMANN, Franz Josef (1979) : «Un dictionnaire de collocations est-il possible ?», TraLiLi, 17-1, pp. 187-195.

HAUSMANN, Franz Josef (1991): «Le dictionnaire de collocations», Wörterbücher, Dictionaries, Dictionnaires, Vol. 3, Berlin, New York, Walter de Gruyter, pp. 1010-1019.

JABOKSON, Roman (1971) : «On Linguistic Aspects of Translation», Selected Writings, The Hague, Mouton.

KUČERA, Antonín, CLAS, André et Jean BAUDOT (1991): Dictionnaire compact des sciences et de la technique, vol. 2, allemand-français, Wiesbaden, Oscar Brandstetter Verlag.

KUČERA, Antonín, ClAS, André et Jean BAUDOT (1995): Dictionnaire compact des sciences et de la technique, vol. 1, français-allemand, Wiesbaden, Oscar Brandstetter Verlag (sous presse).

MELČUUK, Igor (1984) : Dictionnaire explicatif et combinatoire du français contemporain, vol. I, Montréal, Les Presses de l'Université de Montréal.

QUEMADA, Bernard (1972): «Lexicology and Lexicography», Current Trends in Linguistics, Thomas A. Sebeok (Ed.), vol. 9 Linguistics in Western Europe, The Hague, Paris, Mouton, pp. 395-475.

SAUVAGEOT, Aurélien (1964): Portrait du vocabulaire français, Paris, Larousse.

THOIRON, Philippe et Henri BÉJOINT (1989): «Pour un index évolutif et cumulatif de cooccurrents en langue techno-scientifique sectorielle», Meta, 34-4, pp. 661-6671.

WITTFOHT, A. M. (1966) : Dictionnaire technique des matières plastiques, Paris, Eyrolles Éditeur. 